\title{
Design and Analysis of MIMO Multiuser System Using Full-Duplex Multiple Relay Nodes
}

\author{
Tetsuki Taniguchi Yoshio Karasawa \\ Department of Communication Engineering and Informatics \\ Advanced Wireless Communication research Center (AWCC) \\ The University of Electro-Communications (UEC), Tokyo 182-8585, Japan
}

\begin{abstract}
This paper deals with design and analysis of fullduplex relay-aided communication system for the general case of multi-relay and multiuser structure assuming that all of source, relay and destination nodes are equipped with multiple antennas. First, the calculation methods of transmit and receive weight vectors are considered based on zero forcing approach which achieves the cancellation of all the interferences with reduced number of antenna elements. Then computer simulations are carried out to verify the effectiveness of full-duplex relayaided transmission. The results show that the proposed system has better performance compared with relay-less and halfduplex relay-aided cases under certain conditions, and some natures of full-duplex relaying including its disadvantages are also investigated.
\end{abstract}

Index Terms-Relay, full-duplex, MIMO (multiple input multiple output), cooperative communication, zero forcing.

\section{INTRODUCTION}

Wireless communication with relaying is collecting attentions as an attractive choice in high speed and broadband systems [1]-[3] represented by LTE. When the direct path from the source to the destination node is under a bad condition, the wireless transmission aided by relay(s) can improve the link quality and increase the capacity if the connections between source-relay and relay-destination are kept strong.

Normally, as a simple transmission scheme in relay system, the communication is first carried out between source and relay and then relay and destination; by so doing, the influence of the self-interferences in the relay can be avoided. This scheme, called half-duplex relaying, however, reduces the total capacity of the system to the half because of the transmission over two time slots. To avoid such a capacity loss, some papers handle with full-duplex relaying system in which the simultaneous transmission is carried out between source-relay and relay-destination links [5]-[9]. But this scheme requires separation of the receive and transmit antennas of the relay to avoid self-interference, or it brings severe degradation of the communication quality and sometimes results in oscillation phenomenon caused by the closed loop. Ordinarily, the separation of the both antennas is physically constructed, but the enough attenuation of the leakage signal is not easy since they are mounted on the same chassis. Reference [5] has shown the possibility of self-interference mitigation by multiple input multiple output (MIMO) signal processing [4] using multiantenna relay. For single user single relay system, design approaches using zero forcing technique [6] and signal to interference ratio (SIR) maximization method [7] have been proposed, and an information theoretic analysis has been also given by [8]. Lee et al. have extended the problem to two user downlink MIMO relay system based on block diagonalization [9], and shown the potential of full-duplex relaying technique in multiuser system, but because of the consumption of a large number of degrees of freedom (= necessity of many antennas), the system is limited to single relay case where only one of two users can use it.

This paper considers the possibility of full-duplex relaying system for the general case of multiple sources, relays, destinations where all of them are equipped with multiple antennas, and a relay can transact signals of plural users. As the first step, we consider the single stream case, but in multiuser and multi-relay system, in addition to self-interference, the interrelay interference becomes new origin of the performance degradation. Here, to cope with those interferences, a new zero forcing based design approach is considered; this method is suboptimal, but sufficient for our aim, and enables us the evaluation not affected by handling problem of optimization such as the existence of local minima. In addition, since it is adopting engineer-familiar mathematics easy to follow, it can provide many researchers the basis and materials for the development of improved versions. The dominance of full duplex mode might look obvious, but design method using reduced number of antennas is necessary, and the aim of our analysis is to quantify how much improvement (or rather degradation) is derived after consuming so much degrees of freedom for interference cancellation, under the knowledge of perfect channel state information (CSI) (this is an ideal condition but the comparison is fair).

The rest of this paper is organized as follows: after the model of multi-relay system considered in this study is shown in section II, design methods of transmit/receive weight vectors based on zero forcing are investigated in section III. After computer simulations are carried out in section IV to investigate the effectiveness and features of full-duplex relay system, conclusions and future works are described in scetion $\mathrm{V}$.

\section{System Model}

The model of MIMO multi-relay multiuser system considered in this study is depicted in Fig. 1. The system consists of $M$ source nodes expressed by $\mathrm{S}_{0}, \cdots, \mathrm{S}_{M-1}, M_{r}$ relay nodes $\mathrm{R}_{0}, \cdots, \mathrm{R}_{M_{r}-1}$, and $M$ destination nodes $\mathrm{D}_{0}, \cdots, \mathrm{D}_{M-1}$, 

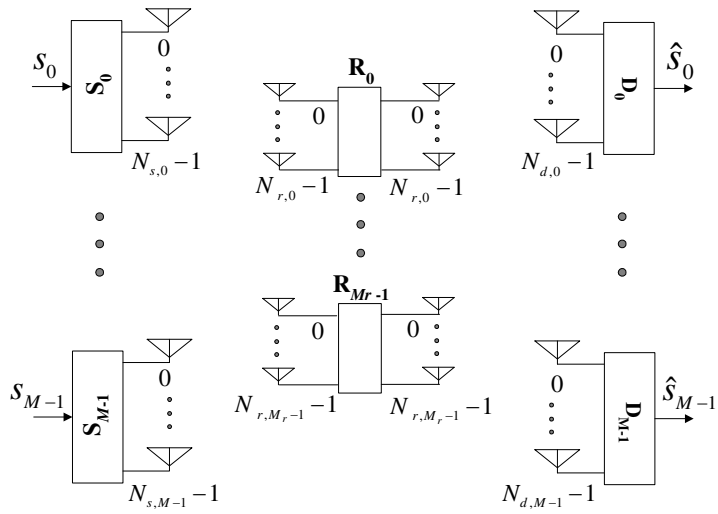

(a) System geometry.

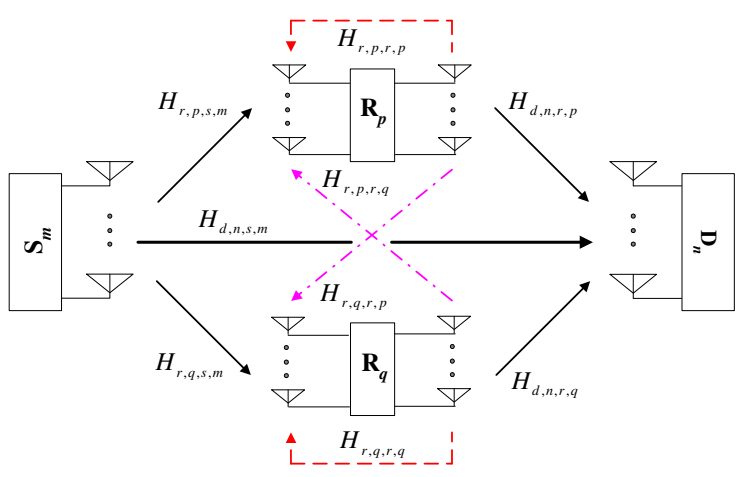

(b) Definition of channels.

Fig. 1. Model of MIMO relay multiuser system consisting of $M$ source nodes $\left(\mathrm{S}_{0} \sim \mathrm{S}_{M-1}\right), M_{r}$ relay nodes $\left(\mathrm{R}_{0} \sim \mathrm{R}_{M_{r}-1}\right)$, and $M$ destination nodes $\left(\mathrm{D}_{0} \sim \mathrm{D}_{M-1}\right)$. A relay can be utilized by plural users. Other than source-destination $\left(H_{d, n, s, m}\right)$, source-relay $\left(H_{r, p, s, m}, H_{r, q, s, m}\right)$, and relay-destination $\left(H_{d, n, r, p}, H_{d, n, r, q}\right)$ channels, in full-duplex relaying, relay self-interference $\left(H_{r, p, r, p}, H_{r, q, r, q}\right)$ and inter-relay interference $\left(H_{r, q, r, p}, H_{r, p, r, q}\right)$ should be considered.

where numbers $m=0, \cdots, M-1$ and $m_{r}=0, \cdots, M_{r}-1$ correspond to user and relay indices, respectively, and each of $\mathrm{S}_{m}, \mathrm{R}_{m_{r}}$ and $\mathrm{D}_{m}$ are equipped with $N_{s, m}, N_{r, m_{r}}$, and $N_{d, m}$ antennas. The MIMO channel between source $S_{m}$ and relay $\mathrm{R}_{n}$ is expressed by $N_{r, n} \times N_{s, m}$ complex matrix $H_{r, n, s, m}$, and other MIMO channels $H_{d, n, r, m} \in \mathbb{C}^{N_{d, n} \times N_{r, m}}$ (relaydestination), $H_{d, n, s, m} \in \mathbb{C}^{N_{d, n} \times N_{s, m}}$ (source-destination), and $H_{r, n, r, m} \in \mathbb{C}^{N_{r, n} \times N_{r, m}}$ (inter-relay) are similarly defined (remark that generally $H_{r, n, r, m}=H_{r, m, r, n}^{T}$ does not consist, since a relay has independent transmit and receive antenna arrays). Source $\mathrm{S}_{m}$ can directly transmit data signal $s_{m}(t)$ to destination $\mathrm{D}_{m}$, but relay-aided communication is also possible; here, the set of relay indices used by $\mathrm{S}_{m}$ is given by $\mathcal{R}_{m}$, and empty $\mathcal{R}_{m}(=\phi)$ means $\mathrm{S}_{m}$ has chosen direct transmission, while notation $\mathcal{R}_{m}^{-1}$ is used to express the set of indices of source nodes which utilize $\mathbf{R}_{m}$.

In full-duplex mode, the data transmission is carried out as exemplified in Fig. 2. If source $S_{m}$ uses relay $\left(\mathcal{R}_{m} \neq \phi\right)$, data $s_{m}(t)$ is transmitted to relay $\left\{\mathrm{R}_{k \in \mathcal{R}_{m}}\right\}$ using weight $\boldsymbol{w}_{s, m, r, k}$, and simultaneously, past data $s_{m}\left(t-t_{d}\right)$ is sent to $\mathrm{D}_{m}$ after the multiplication of weight $\boldsymbol{w}_{s, m, d, m}$. If source $\boldsymbol{S}_{m}$ does not use relay $\left(\mathcal{R}_{m}=\phi\right)$, weight $\boldsymbol{w}_{s, m, r, k}$ is not used, and only current data $s_{m}(t)$ is transmitted to the destination using weight $\boldsymbol{w}_{s, m, d, m}$. The relay $\mathrm{R}_{k}$ receives the signal from $\left\{\mathrm{S}_{m \in \mathcal{R}_{k}^{-1}}\right\}$ using weight $\boldsymbol{w}_{r, r, k, m}$, and the relay output signal $\hat{s}_{r, k, m}(t)$ which is replica of $s_{m}(t)$ is produced. The signal transmitted from relay $\mathrm{R}_{k}$ to destination $\mathrm{D}_{m}$ is $s_{r, k, m}(t)=\mathcal{P} \hat{s}_{r, k, m}(t)$, where operator $\mathcal{P}$ works as $\mathcal{P} x=x$ (namely, $s_{r, k, m}(t)=$ $\hat{s}_{r, k, m}(t)$ ) if AF relaying is used, and the decoded (then error corrected and remodulated) version of $\hat{s}_{r, k, m}\left(t-t_{d}\right)$ (namely, $s_{r, k, m}\left(t-t_{d}\right)=s_{m}\left(t-t_{d}\right)$ when there's no error) is generated by $\mathcal{P}$ if DF relaying is adopted; for both cases, weight vector $\boldsymbol{w}_{r, t, k, m}$ is multiplied before sending the data. In the relay aided link, since signal $s_{m}\left(t-t_{d}\right)$ is sent simultaneously from $\mathrm{S}_{m}$ and $\left\{\mathrm{R}_{k \in \mathcal{R}_{m}}\right\}$, weights $\boldsymbol{w}_{s, m, d, m}$ and $\left\{\boldsymbol{w}_{r, t, k, m}\right\}$ could be designed assuming cooperative transmission among them, though we should remark that what is sent from relays is noise corrupted version of $s_{m}\left(t-t_{d}\right)$ if AF scheme is adopted.

In half-duplex mode, in the first phase, the transmission of $s_{m}(t)$ to destination $\mathrm{D}_{m}$ and relays $\left\{\mathrm{R}_{k \in \mathcal{R}_{m}}\right\}$ (if $\mathcal{R}_{m} \neq \phi$ ) is carried out using weights $\boldsymbol{w}_{s, m, d, m}$ and $\boldsymbol{w}_{s, m, r, k}$, respectively. Then in the second phase, source $S_{m}$ transmits $s_{m}(t+1)\left(\mathcal{R}_{m}=\phi\right)$ or retransmits $s_{m}(t)\left(\mathcal{R}_{m} \neq \phi\right)$ using weight $\boldsymbol{w}_{s, m, d, m}$, and relay $\mathbf{R}_{k}$ transmits $s_{r, k, m}(t)$ using weight $\boldsymbol{w}_{r, t, k, m}$. Also here, the cooperative transmission of $\mathbf{S}_{m}$ and $\left\{\mathbf{R}_{k \in \mathcal{R}_{m}}\right\}$ is possible.

All the weights should be designed attempting the interference cancellation against nontarget receivers and strong connection toward the target destination. In the next section, actual procedure of weight calculation is considered based on zero forcing.

\section{Design Method}

In this section, the design problem of five types of weights $\left(\boldsymbol{w}_{s, m, d, m}, \boldsymbol{w}_{s, m, r, k}, \boldsymbol{w}_{r, r, k, m}, \boldsymbol{w}_{r, t, k, m}, \boldsymbol{w}_{d, m}\right)$ in the model of the previous section is studied. Here we consider the downlink transmission, and assume conditions $N_{s, m} \geq N_{r, k}$ and $N_{r, k} \geq N_{d, m}\left({ }^{\forall} m \in \mathbb{Z}[0, M-1],{ }^{\forall} k \in \mathbb{Z}\left[0, M_{r}-1\right]\right)$, but the idea could be easily applied to other cases such as uplink. All the methodologies mentioned below include zero forcing process, but the term 'zero forcing' in the subsection title is used for the discrimination of those methods from block diagonalization (BD) [11] for the convenience of naming. Here, for a set $\mathcal{X}=\left\{X_{0}, \cdots, X_{N-1}\right\}$, notation $[\mathcal{X}]=\left[X_{0}^{T}, \cdots, X_{N-1}^{T}\right]^{T}$ means the stacking of all the elements of set $\mathcal{X}$ in raw (similarly, $[\mathcal{X}, \mathcal{Y}]^{T}=$ $\left.\left[X_{0}^{T}, \cdots, X_{M-1}^{T}, Y_{0}^{T}, \cdots, Y_{N-1}^{T}\right]^{T}\right)$, and another expression $\tilde{H}_{d, m, r, \mathcal{R}_{m}}$ denotes the column-wise stacking of all the elements of set $\left\{H_{d, m, r, k}, k \in \mathcal{R}_{m}\right\}$. Notation $\mathcal{N}$ denotes the vector normalization operator, namely, $\mathcal{N} \boldsymbol{x}=\boldsymbol{x} /\|\boldsymbol{x}\|$.

\section{A. Full-Duplex BD Design}

A straight way of multi-relay weight design in MIMO case is the extension of BD based method (e.g., in [9]); for 

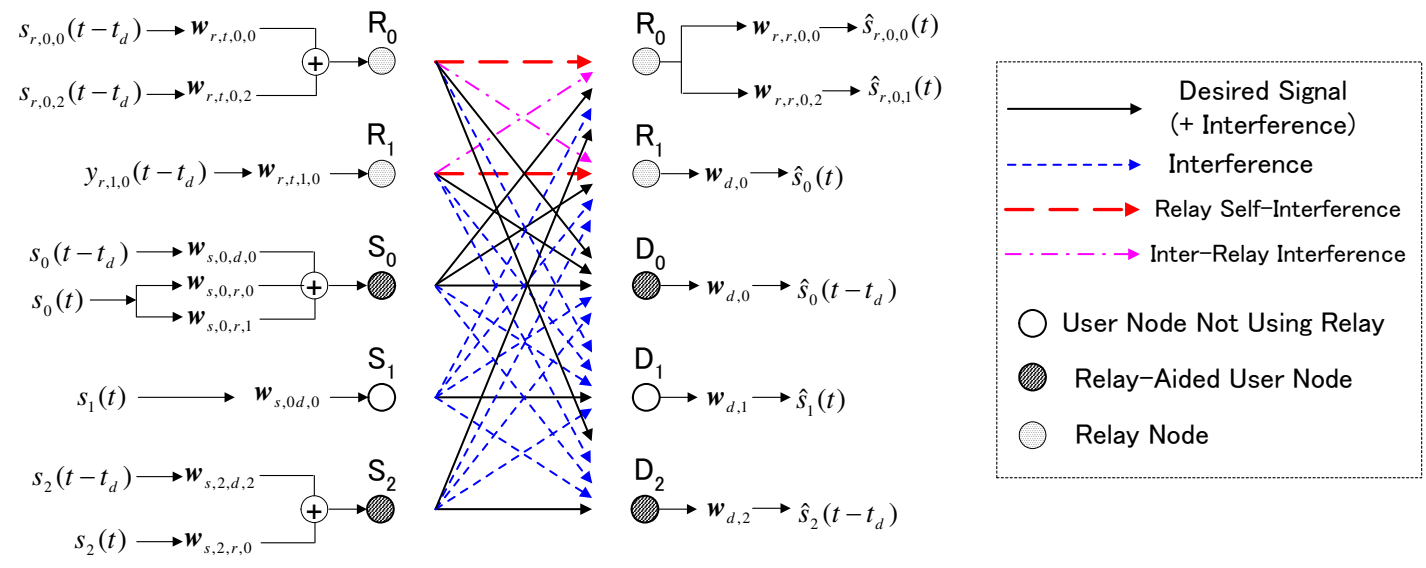

Fig. 2. Example of signal flow diagram in case of $M=3, M_{r}=2, \mathcal{R}_{0}=\{0,1\}, \mathcal{R}_{1}=\phi$, and $\mathcal{R}_{2}=\{0\}$. Node $\mathrm{R}_{m}(m=0,1)$ in left and right sides shows the transmitter and receiver aspects of same relay node. Other than three types of dashed lines, some of solid lines $\left(S_{0}-D_{0}, S_{0}-R_{0}, S_{0}-\right.$ $R_{1}, S_{2}-D_{2}, S_{2}-R_{0}, R_{0}-D_{0}, R_{0}-D_{2}$ ) also contain interference components, and they should be eliminated by array processing.

example, $\boldsymbol{w}_{s, m, r, k}$ and $\boldsymbol{w}_{r, r, k, m}$ can be designed applying BD to multiuser MIMO channel

$\left[H_{r, 0, s, m}^{T}, \cdots, H_{r, M_{r}-1, s, m}^{T}, H_{d, 0, s, m}^{T}, \cdots, H_{d, M-1, s, m}^{T}\right]^{T}$, where the target channel is $H_{r, k, s, m}$. In the same manner, weights $\boldsymbol{w}_{s, m, d, m}, \boldsymbol{w}_{r, t, k, m}$, and $\boldsymbol{w}_{d, m}$ can be derived from multiuser MIMO channel

$\left[H_{d, 0, r, m}^{T}, \cdots, H_{r, M_{r}-1, s, m}^{T}, H_{d, 0, s, m}^{T}, \cdots, H_{d, M-1, s, m}^{T}\right]^{T}$, where the target channel is $H_{r, k, s, m}$.

In this approach, one degree of freedom is necessary for the null steering to each antenna of nontarget relays and destinations. Therefore, in multi-relay multiuser multiantenna system handled in this study, the total number of degrees of freedom, namely, minimum antenna number of source and relay becomes very large. More fatally, if one relay node is utilized by plural sources, the relay cannot separate the signals from different sources using the weights designed by the conventional BD. For those reasons, the use of BD in our system assumption is impractical and/or impossible, so this method is excluded from our choice.

\section{B. Full-Duplex Zero Forcing Design}

In this method, to reduce the consumption of the degrees of freedom used in zero forcing, the weights of one of transmitter or receiver sides are first determined in each of sourcedestination, source-relay, relay-destination links, then those of other side are calculated based on zero forcing. Consequently, in this strategy, the design order of the previously mentioned five weights has importance, hence in this paragraph, we will discuss this problem. To utilize the large number of antennas installed in the source, it is preferred that weights $\boldsymbol{w}_{s, m, d, m}$ and $\boldsymbol{w}_{s, m, r, k}$ are designed in the last step (weights $\boldsymbol{w}_{s, m, d, m}$ and $\boldsymbol{w}_{s, m, r, k}$ can determined independently, hence their design order is not a problem). Since the destinations which are usually equipped with small number of antennas have poor ability of zero forcing, it is also desirable that $\boldsymbol{w}_{d, m}$ is calculated first of all, not attempting interference cancellation. In case of relay, the degrees of freedom in transmit and receive weights are same, but taking account the possibility of sourcerelay cooperative transmission, relay transmit weight $\boldsymbol{w}_{r, t, k, m}$ should be designed together with the source weight $\boldsymbol{w}_{s, m, d, m}$ after deriving receive weight $\boldsymbol{w}_{r, r, k, m}$.

According to the above design order, actual desigen is carried out as follows:

(1) Design of $\boldsymbol{w}_{d, m}$ and $\boldsymbol{w}_{r, r, k, m}$

Derivation of those weights which optimise a certain criterion simultaneously achieving the zero forcing is not a tractable problem. Therefore, attempting to establish a good connection with the transmitter side, we choose singular value decomposition (SVD) as the second best; namely, by defining matrix $H_{d, m, s, r, \mathcal{R}_{m}}=\left[\begin{array}{lll}H_{d, m, s, m} & \tilde{H}_{d, m, r, \mathcal{R}_{m}}\end{array}\right] \in$ $\mathbb{C}^{N_{d, m} \times\left(N_{s, m}+N_{r, \mathcal{R}_{m}}\right)}$, where $N_{r, \mathcal{R}_{m}}=\sum_{k \in \mathcal{R}} N_{r, k}$, weights $\boldsymbol{w}_{d, m}$ and $\boldsymbol{w}_{r, r, k, m}$ are respectively derived as the left singular value vectors of $H_{d, m, s, r, \mathcal{R}_{m}}$ and $H_{r, k, s, m}$ corresponding to the largest singular value of each matrix.

(2) Design of $\boldsymbol{w}_{s, m, r, k}$

Define a zero forcing matrix $H_{z f, s, m}$ as follows:

$H_{z f, s, m}=\left[\begin{array}{cc}\left\{\boldsymbol{w}_{r, r, \ell, p}^{H} H_{r, \ell, s, m} ;\right. & \ell \in \mathbb{Z}\left[0, M_{r}^{-1}-1\right]\end{array}\right\}$

Weight $\boldsymbol{w}_{s, m, r, k}$ could be derived by applying zero forcing to $H_{z f, s, m}$ setting the target matrix (though it is actually a raw vector) as $\boldsymbol{w}_{r, r, k, m}^{H} H_{r, k, s, m}$. Namely, $\boldsymbol{w}_{s, m, r, k}=$ $\mathcal{N} V_{s, m, r, k} V_{s, m, r, k}^{H} H_{r, k, s, m}^{H} \boldsymbol{w}_{r, r, k, m}$, where $V_{s, m, r, k}$ is a matrix 
whose column vectors span the null space of

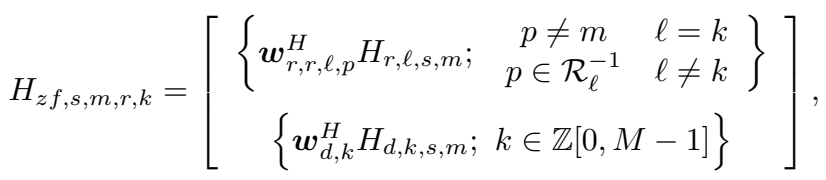

which is derived by zero forcing. By this operation, relay $\mathrm{R}_{k}$ can extract the signal from $\mathbf{S}_{m}$ by using $\boldsymbol{w}_{r, r, k, m}$.

(3) Design of $\boldsymbol{w}_{s, m, d, m}$ and $\boldsymbol{w}_{r, t, k, m}$

To achieve cooperative transmission of $S_{m}$ and relays $\left\{\mathrm{R}_{k \in \mathcal{R}_{m}}\right\}$, set of weights $\left\{\boldsymbol{w}_{s, m, d, m}, \boldsymbol{w}_{r, t, k, m} ; k \in \mathcal{R}_{m}\right\}$ forms a virtual large-size array.

In the zero forcing operation to this matrix, we have three options which become important particularly in case of AF relaying, since all the members of virtual array may have differently noise corrupted signal [10]; that is, the source has noise free signal while the different noise components (and residual interference component if the zero forcing is not perfect) are contained in the output signals of different relays ${ }^{1}$. The straight way is the zero forcing by virtual weight $\boldsymbol{w}_{s, r, m}=\left[\boldsymbol{w}_{s, m, d, m}^{T},\left[\left\{\boldsymbol{w}_{r, t, k, m} ; k \in \mathcal{R}_{m}\right\}\right]^{T}\right]^{T}$ (Type 1). In this case, the degrees of freedom are large, but instead, the leakage undesired components might be generated in zero forcing process by dealing differently noise-corrupted signals as the same one, and degrade the interference cancellation ability. The second choice is independent zero forcing for the noise-less and noise corrupted signals, namely, $\boldsymbol{w}_{s, m, d, m}$ and $\left[\left\{\boldsymbol{w}_{r, t, k, m} ; k \in \mathcal{R}_{m}\right\}\right]$ separately carry out zero forcing (Type 2). The third one is separate zero forcing of each of the source and relays, one by one (Type 3). Type 3 could achieve leakage-less zero forcing, but it requires large number of antennas for each of source and relays, namely, $\min \left\{N_{s, m}, N_{r, k} ; k \in \mathcal{R}_{m}\right\}>M-2+\sum_{k=0}^{M_{r}-1}\left|\mathcal{R}_{k}^{-1}\right|$ should be satisfied, hence the condition of application becomes severer.

Here, to investigate the ability of full-duplex relay under the existence of leakage component, we dare to choose Type 2 for DF even if the source and relays have sufficient number of antennas for adopting Type 3 scheme with a better performance (to avoid the oscillation, we used Type 3 for AF case). In this case, the zero forcing matrices are $H_{z f, s, m}$ and $H_{z f, r, \mathcal{R}_{m}}$, where $H_{z f, r, \mathcal{R}_{m}}$ is defined as

$H_{z f, r, \mathcal{R}_{m}}=\left[\begin{array}{cc}\left\{\boldsymbol{w}_{r, r, \ell, p}^{H} \tilde{H}_{r, \ell, r, \mathcal{R}_{m}} ;\right. & \ell \in \mathbb{Z}\left[0, M_{r}-1\right] \\ \left\{\boldsymbol{w}_{d, k}^{H} \tilde{H}_{d, k, r, \mathcal{R}_{m}} ;\right. & k \in \mathbb{Z}[0, M-1]\}\end{array}\right]$

and target matrices (vectors) are $\boldsymbol{w}_{d, m}^{H} H_{d, m, s, m}$ and $\boldsymbol{w}_{d, m}^{H} \tilde{H}_{d, m, r, \mathcal{R}_{m}}$, respectively. In a similar manner as

${ }^{1}$ Also in case of DF relaying, the noise may result in the error of the decoded signal in one (or some) of relay(s), which means different transmit signals are sent from different relays. But here, we assume the error correction is well working in DF relays, and their transmit signal contains no error. the derivation of $V_{s, m, r, k}$ in (2), null space matrices derived in the zero forcing process are denoted by $V_{s, m, d, m}$ and $V_{r, t, \mathcal{R}_{m}}$, then a raw vector $\boldsymbol{h}=$ $\left[\boldsymbol{w}_{d, m}^{H} H_{d, m, r, m} V_{s, m, d, m}, \boldsymbol{w}_{d, m}^{H} \tilde{H}_{d, m, r, \mathcal{R}_{m}} V_{r, t, \mathcal{R}_{m}}\right]$ is calculated, and finally $\boldsymbol{w}_{s, m, d, m}=V_{s, m, d, m} \boldsymbol{h}^{H} /\|\boldsymbol{h}\|$ and $\left[\left\{\boldsymbol{w}_{r, t, k, m} ; k \in \mathcal{R}_{m}\right\}\right]=V_{r, t, \mathcal{R}_{m}} \boldsymbol{h}^{H} /\|\boldsymbol{h}\|$ are derived, (therefore, the signal combining operation is always accomplished cooperatively by source and relay weights).

\section{Half-Duplex Zero Forcing Design}

The calculation method of half-duplex weights is easily derived by modifying that of the full-duplex relay system described in the previous section. Design method of $\boldsymbol{w}_{d, m}$ and $\boldsymbol{w}_{r, r, k, m}$ is same as section III-B. Weight $\boldsymbol{w}_{s, m, r, k}$ can be designed by replacing $H_{z f, s, m}$ as follows:

$$
\begin{aligned}
& H_{z f, s, m}= \\
& {\left[\begin{array}{cc}
\left\{\boldsymbol{w}_{r, r, \ell, p}^{H} H_{r, \ell, s, m} ;\right. & \left.\begin{array}{c}
p \in \mathcal{R}_{\ell}^{-1} \\
\mathbb{Z}\left[0, M_{r}-1\right]
\end{array}\right\} \\
\left\{\boldsymbol{w}_{d, k}^{H} H_{d, k, s, m} ; k \in \mathbb{Z}[0, M-1] \text { s.t. } \mathcal{R}_{k}=\phi\right\}
\end{array}\right] .}
\end{aligned}
$$

Weights $\boldsymbol{w}_{s, m, d, m}$ and $\left[\left\{\boldsymbol{w}_{r, t, k, m} ; k \in \mathcal{R}_{m}\right\}\right]$ are derived in the similar way using zero forcing matrices $H_{z f, s, d, m}$ and $H_{z f, r, t, m}$ given by

$$
\begin{aligned}
& \left.H_{z f, s, d, m}=\left[\left\{\boldsymbol{w}_{d, k}^{H} H_{d, k, s, m} ; k \in \mathbb{Z}[0, M-1] \backslash\{m\}\right\}\right\}\right] \\
& H_{z f, r, \mathcal{R}_{m}}=\left[\left\{\boldsymbol{w}_{d, k}^{H} H_{d, k, r, \mathcal{R}_{m}} ; k \in \mathbb{Z}[0, M-1] \backslash\{m\}\right\}\right]
\end{aligned}
$$

(the operatin after zero forcing is same as the full-duplex case).

By using half-duplex mode, usually smaller number of degrees of freedom is required to realize zero forcing, hence normally it brings higher quality of output signal compared with full-duplex case. On the other hand, since the transmission is over two time slots, the resultant total capacity is decreased.

\section{COMPuter Simulation AND Discussion}

This section describes computer simulation to verify the effectiveness and natures of the proposed full-duplex relay system. The default simulation conditions used in this study are summarized in Table I.

The user number is $M=3$, where all users initially attempt using two relays. Normally, different from destination nodes which are assumed as mobile terminals, relays are fixed on a line-of-sight (LOS) position to keep a strong connection with the target source node as possible (though in some applications, relays might be obliged to be placed on a non LOS position). Taking into account this fact, in this paper, the variation of source-relay channels is simulated by Rician fading (Rician factor $K=3$ ). The sourcedestination and relay-destination channels are assumed to be under i.i.d. (independent and identically distributed) Rayleigh fading, and here, the source-destination link (the variance of each matrix element is $\left.\sigma^{2}=0.3\right)$ is weaker than that of the relay-destination $\left(\sigma^{2}=1\right)$. The definition of inter-relay channels is not easy like others; relays are usually fixed and 
TABLE I

Simulation CONDITIONS.

\begin{tabular}{|c|c|}
\hline Number of Users & $M=3$ \\
\hline Number of Relays & $\begin{array}{c}M_{r}=1 \sim 4 \\
\left(\text { default }: M_{r}=3\right)\end{array}$ \\
\hline Relay Selection & $\mathcal{R}_{0}=\{0,1\}, \mathcal{R}_{1}=\{1,2\}, \mathcal{R}_{2}=\{2,0\}$ \\
\hline Antenna Number & $\left(N_{s, m}, N_{r, m}, N_{d, m}\right)(10,10,2)$ \\
\hline Modulation & QPSK \\
\hline Relaying Scheme & AF or DF \\
\hline Zero Forcing & Type 2 (DF) Type 3 (AF) \\
\hline Relay SNR & SNR $_{r, m}=5 \sim 30 \mathrm{~dB}$ \\
(default : 20dB)
\end{tabular}

the channels may not be deeply faded, and the self-interference might be stronger than inter-relay interference because the distance between the transmit and receive antennas is shorter compared with the inter-relay case. In spite of those facts, here, the simulation is carried out assuming all of them are i.i.d. Rayleigh faded, because it can represent the channel variation by the change of the locations of multiple relays through the randomly generated matrices (we have actually verified for the various fixed relay case that the total conclusion is same as shown below) ${ }^{2}$. In addition, the relay station is physically designed to mitigate the self-interference, hence we consider it is weaken to a level comparable to inter-relay interferences.

The metric of the performance evaluation is capacity calculated by $C_{m}=\log _{2}\left(1+\Gamma_{m}\right)$ for User $m$, where $\Gamma_{m}$ is signal to noise plus interference ratio (SINR) at destination node $\mathrm{D}_{m}$. The signal to noise ratios (SNRs) in relays and destinations are defined as $\mathrm{SNR}_{r, m}=P_{S . m} / P_{N, r, m}$ and $\mathrm{SNR}_{d, m}=P_{S . m} / P_{N, d, m}$, where $P_{S, m}=E\left[\left|s_{m}(t)\right|^{2}\right]$, $P_{N, r, m}$, and $P_{N, d, m}$ denote signal power, relay and destination noise powers, respectively. The value of $t_{d}>0$ affects the delay of the data reception, but does not concern the performance.

The effectiveness of relay processing is influenced by the relay selection scheme and criterion for the switching between direct and relay-aided transmissions. In this paper, to avoid the complexity of relay selection which is affected by the setting of various parameters, all the users first attempt to use the relay, but if SINR of the received signal of user $m$ at relay $\mathrm{R}_{k}$ is less than $\operatorname{SINR}_{r, d}=12 \mathrm{~dB}$ after the weight design, $k$ is removed from $\mathcal{R}_{m}$, then weights are recalculated (this operation is repeated with the variation of the channel, and requires a larger overhead as the condition of relay-aided channel becomes worse). Without this procedure, the relays in bad condition may chosen, and it brings not only the performance degradation but also the error of the

${ }^{2}$ Recently, Softbank Telecom, Japan, has developed temporal relay station installed on balloon (InfoBalloon) moored to a vehicle for the quick recovery from a disaster [12]. If this type of relay system is used in our model, channel $H_{r, n, r, m}$ could be suffered from fading due to the welter effect by wind, but it is not yet clear whether the fading type is Rician or not.

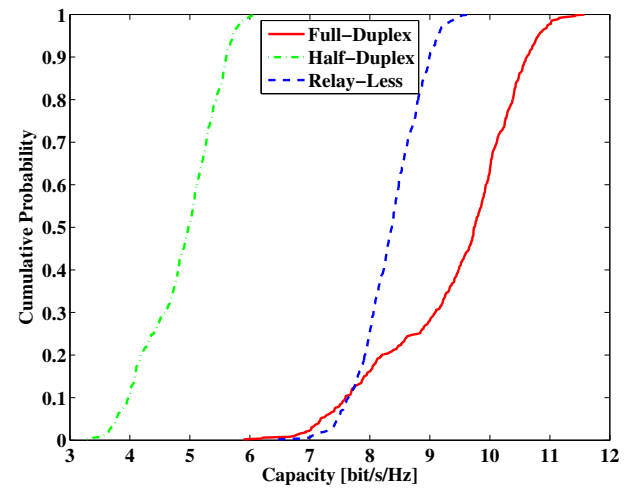

(a) $\mathcal{R}_{0}=\{0,1\}, \mathcal{R}_{1}=\{1,2\}, \mathcal{R}_{2}=\{2,0\}, \mathrm{SNR}_{r, m}=20 \mathrm{~dB}$.

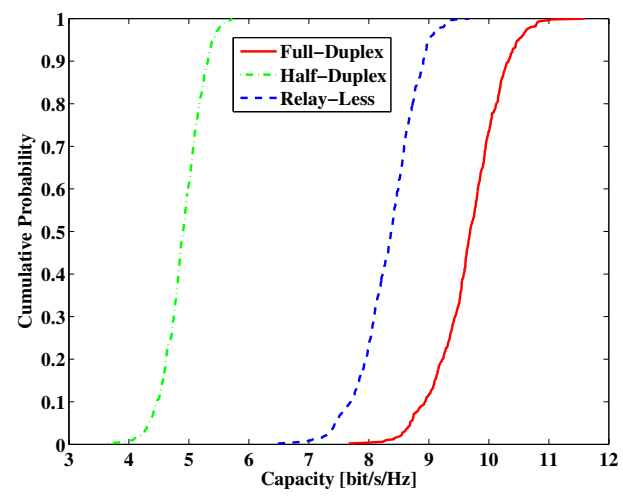

(b) $\mathcal{R}_{0}=\{0\}, \mathcal{R}_{1}=\{1\}, \mathcal{R}_{2}=\{2\}, \mathrm{SNR}_{r, m}=20 \mathrm{~dB}$.

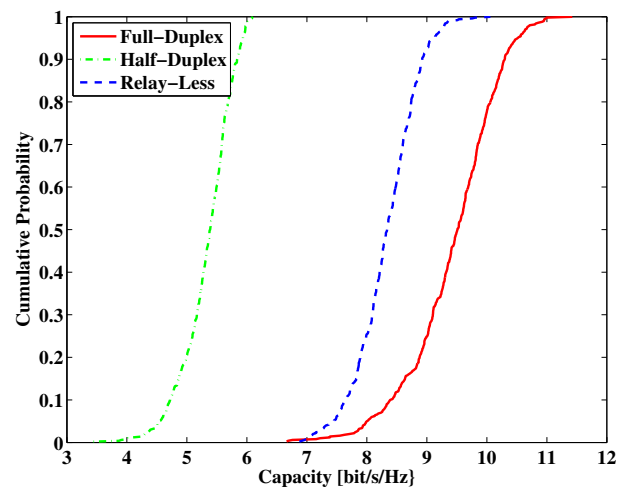

(c) $\mathcal{R}_{0}=\{0,1\}, \mathcal{R}_{1}=\{1,2\}, \mathcal{R}_{2}=\{2,0\}, \mathrm{SNR}_{r, m}=30 \mathrm{~dB}$.

Fig. 3. Distribution functions of capacity of User 0 in DF relaying.

relay data (DF) or oscillation by the feed back through selfinterference (AF), hence the direct/relay-aided link switching has a particular importance in full duplex system. The best is relay selection based on the channel condition, but here to keep the problem setting simple, such a dynamic relay allocation is not considered.

Let us start the simulation from the DF relaying case. Figure 3 depicts distribution functions of capacity of User 0 (curves 


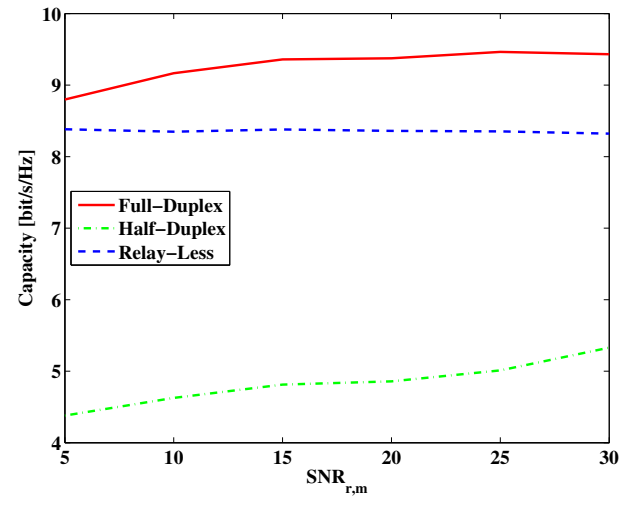

Fig. 4. Relay SNR versus output SINR of User 0 in DF relaying.

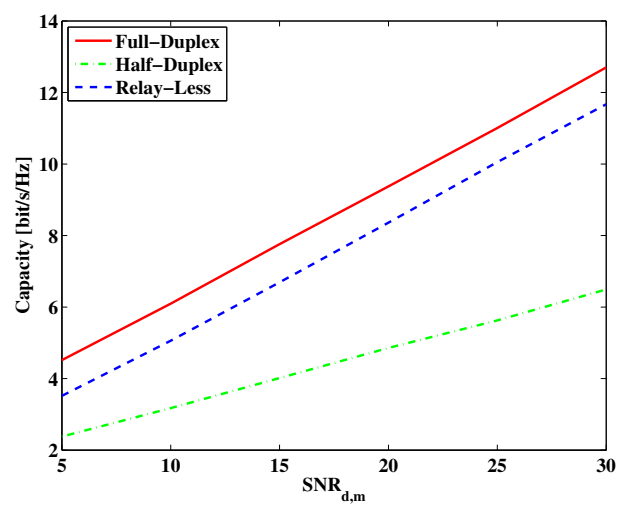

Fig. 5. Destination SNR versus output SINR of User 0 in DF relaying.

of all users almost coincide due to the symmetry of the system, hence those of other users are not given here) for three different transmission strategy. In all of them, the proposed full-duplex system has a better performance than other two approaches. Under this condition, the performance of halfduplex relaying is worse than the relay-less direct transmission. Comparing subplots (a) and (b), while entire curve of full-duplex system is located on the right side of relay-less scheme in (b), the lower edge of full-duplex curve is extended to the left of relay-less one, which means the outage capacity of the proposed method is lower if a relay is shared by plural users. This situation is improved by using a relay with higher SNR, by which full-duplex relay outperforms relay-less scheme also in the point of outage characteristic (we consider this problem is ascribed to the selectin process of relay-aided and direct transmissions, and the investigation on the way to improve this disadvantage without increasing the relay SNR is now underway, but it is not clear at this moment that it can overcome the case without relay-sharing in (b)).

Figure 4 plots the relation between relay SNR and capacity keeping the destination SNR to $20 \mathrm{~dB}$. Though DF relays are adopted and the noise components are eliminated from the transmit data of relays, the performance is influenced by SNR

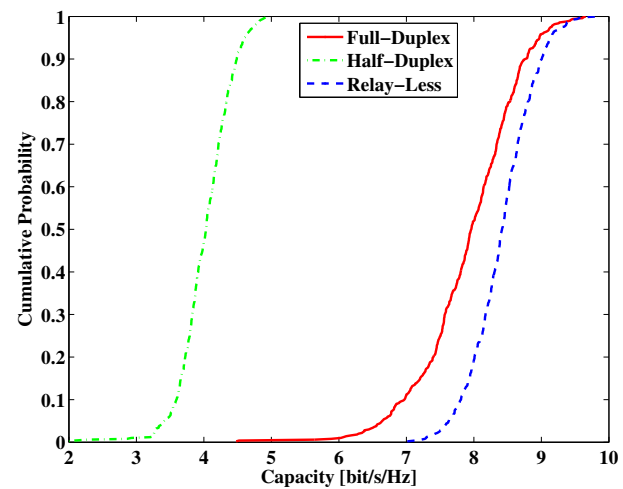

(a) Variance $\sigma^{2}=0.3$ for $H_{d, n, s, m}$.

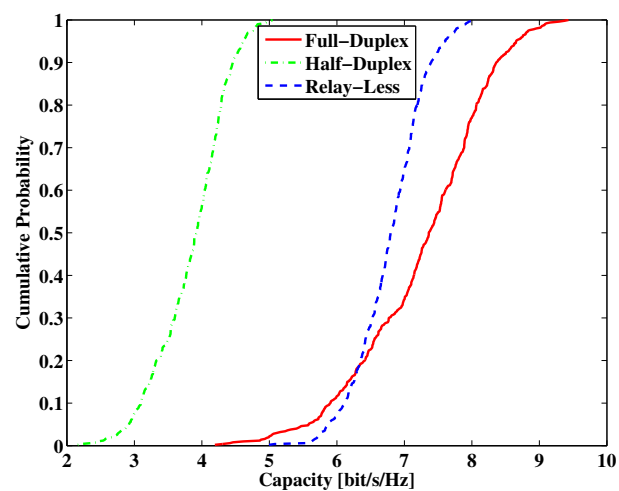

(b) Variance $\sigma^{2}=0.1$ for $H_{d, n, s, m}$.

Fig. 6. Distribution functions of capacity of User 0 in AF relaying.

since this value affects the switching between the relay-aided and direct transmissions. The curve of relay-less scheme is constant because it does not use relay and $\mathrm{SNR}_{r, m}$ does not affect the performance. From this figure, it can be observed that the capacity of full-duplex relay compared with relay-less transmission becomes larger as relay SNR improves; together with the result of Fig. 3, this fact shows the necessity of using high SNR relay. Of course, even if the value of $\mathrm{SNR}_{r, m}$ is increased, the capacity is saturated by the destination SNR, as observed in Fig. 4.

Figure 5 draws the curve of destination SNR versus capacity under the relay SNR of 20dB. The capacity improves as destination SNR increases, and utilization of DF strategy (relays can transmit noise-less data) prevents that the performance is easily limited by relay SNR like the case of AF relaying.

Next, the target is moved to AF relays. Figure 6 (a) denotes the distribution functions of capacity of User 0 , where different from DF case, the performance of full-duplex AF relaying is worse than that of relay-less transmission. This is because in $\mathrm{AF}$ scheme, the noise generated at the relay is transmitted as it is, and it results in the bad quality of the output signal. But if the source-destination link becomes weaker and channel has a variance of 0.1 , AF relay scheme has a chance to be better 


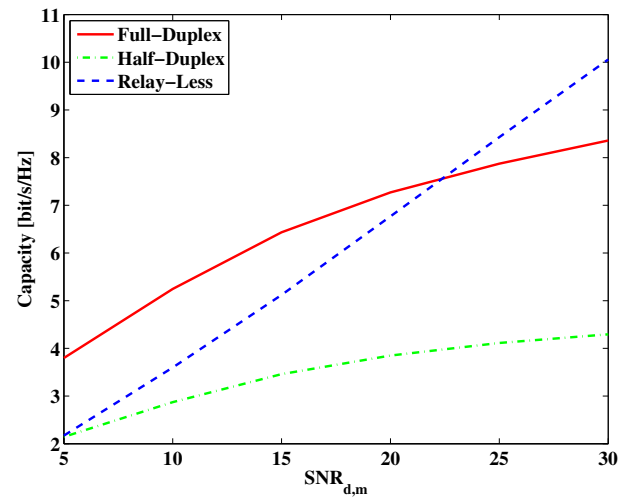

Fig. 7. Destination SNR versus capacity of User 0 in AF relaying.

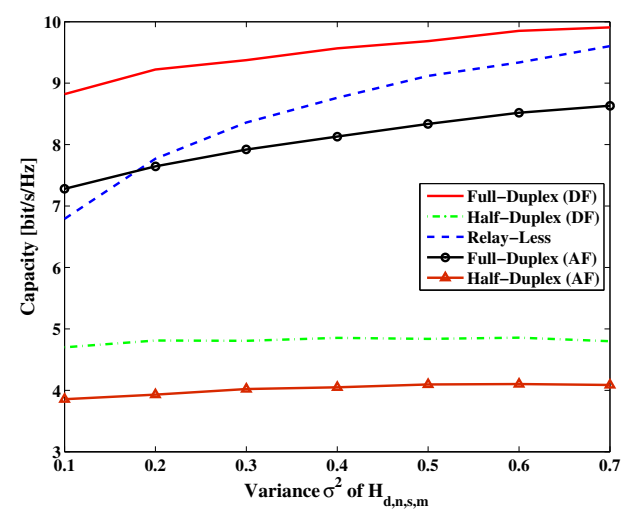

Fig. 8. Variance $\sigma^{2}$ of $H_{d, n, s, m}$ and capacity of User 0 .

choice as shown in Fig. 6 (b).

Figure 7 is an AF version of Fig. 5, namely, it shows the relation between destination SNR and capacity under the relay SNR of $20 \mathrm{~dB}$. Considering that the noise effect is larger in $\mathrm{AF}$ relaying, $\mathrm{SINR}_{r, d}$ is changed to $15 \mathrm{~dB}$, so that the relation between AF relay-aided and relay-less systems becomes more clear. Also in this scheme, the capacity increases as destination SNR becomes higher, but different from DF case, it is saturated in high SNR region by the effect of noise corrupted dirty relay transmit signal.

The capacity with respect to variance $\sigma^{2}$ of $H_{d, n, s, m}$ is drawn in Fig. 8 for both $\mathrm{DF}$ and $\mathrm{AF}$ cases. It is clear that the advantage of relaying is decreased as direct connection between source and destination becomes stronger. Particularly, $\mathrm{AF}$ is effective only when $\sigma^{2}$ is small.

Figure 9 shows how the capacity of User 0 changes as the number of relay is increases. The curves are plotted for three cases, where a relay is shared by one, two, or three users (e.g., for $M_{r}=3, \mathcal{R}_{m}=\{0,1,2\}$ for all $m$ ). In two and three user cases, the maximum number of relay becomes three and two because of the limitation of the degrees of freedom (antenna numbers of source and relay). The capacity becomes larger as the number of relays is increased; the improvement seems to

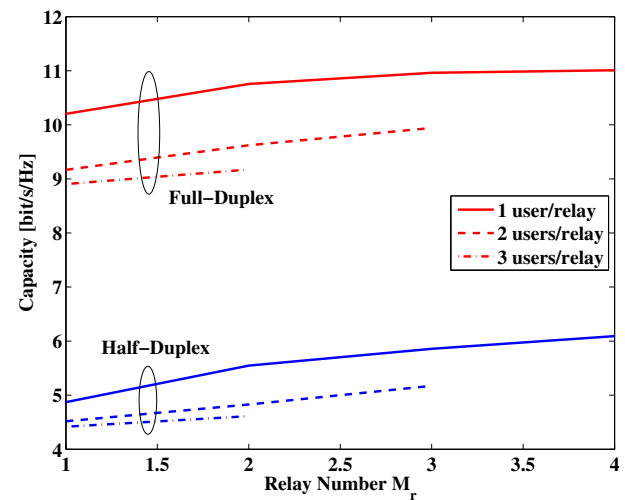

Fig. 9. Relay number versus capacity of User 0 in DF relaying; each relay is shared by one, two, or three users.

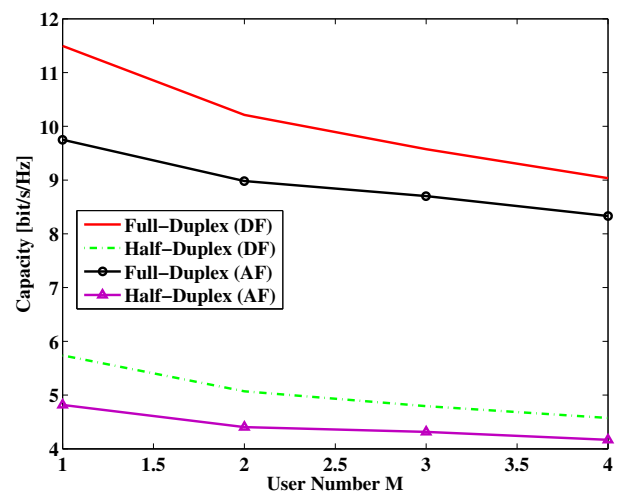

Fig. 10. User number versus capacity of User $0\left(M_{r}=2, \mathcal{R}_{m}=\{0,1\}\right)$.

be not significant in our simulation condition in which all the source-relay and relay-destination channels are homogeneous, but we should remark that this graph is drawn for User 0 ; if a relay is shared by plural users, the capacity of all of them, namely, the sum capacity is increased.

Figure 10 depicts user number versus capacity curves in two relay system $\left(M_{r}=2\right.$ but relay antenna number is increased to $N_{r, 0}=N_{r, 1}=12$ to derive enough degrees of freedom) for both of DF and AF relaying when all users use all relays $\left(\mathcal{R}_{m}=\{0,1\}\right)$. The performance degrades as the user number increases, and the difference between DF and AF becomes smaller because the choice of the relay-aided link is decreased because of the degradation of SINR in relay.

From the results of this section, we can conclude that the full-duplex multi-relay aided transmission has an advantage in performance upgrading of multiuser MIMO system, but it is also clear that it still has some problems and shortages which need to be further improved (e.g., it requires larger number of antennas for source and relay nodes, larger overhead for the weight reculculation to avoid the oscillation, and larger computational cost). 


\section{CONCLUSION}

This paper has considered the design and analysis of fullduplex multi-relay MIMO multiuser system. First, zero forcing based weight calculation approaches for single stream transmission have been proposed to make possible the full-duplex relay design with reduced antenna number. The effectiveness of full-duplex relay has been investigated through computer simulations. Throught those analyses, it has been shown that the proposed system can achieve better performance than half-duplex relay-aided and relay-less schemes under certain conditions. Particularly, the capacity of the full duplex system is nearly twice as much as that of half duplex case, in spite of the consumption of a large number of degrees of freedom for the interference cancellation.

The future subject is the extension of this work to multistream transmission and the application to actual communication system including more detailed resource allocation and relay selection scheme. The measurement campaign of multirelay system depending on applications to know the nature of channel concerning relays is another attractive theme of the study.

\section{ACKNOWLEDGMENT}

The authors would like to thank to Dr. Teruya Fujii, Softbank Telecom, Japan, for providing the information of InfoBalloon, and are grateful to Dr. Nordin Bin Ramli, MIMOS Berhad, Malaysia.

\section{REFERENCES}

[1] X. Tao, X. Xu, and Q. Cui, "An overview of cooperative communications," IEEE Commun. Mag., vol.50, no.6, pp.65-71, June 2012.

[2] G. Kramer et al. ed., Special issue on models, theory and codes for relaying and cooperation in communication networks, IEEE Trans. Inf. Theory, vol.53, no.10, Oct. 2007.

[3] A. Ben Nacef, S.-M. Senouci, Y. Ghamri-Doudane, and A.-L. Beylot, "Enhanced relay selection decision for cooperative communication in energy constrained networks," 2009 2nd IFIP Wireless Days (WD2009), Paris, France, Dec. 2009.

[4] A. Sibille, C. Oestges, and A. Zanella, MIMO : from theory to implementation, Elsevier, Burlington, MA, 2011.

[5] D. W. Bliss, P. A. Parker, and A. R. Margetts, "Simultaneous transmission and reception for improved wireless network performance," Proc. IEEE 14th Workshop Statistical Signal Process. 2007 (SSP '07), Madison, WI, Aug. 2007.

[6] B. Chun, H. Park, "A spatial-domain joint-nulling method of selfinterference in full-duplex relays," IEEE Commun. Lett., vol.16, no.4, pp.436-438, Apr. 2012.

[7] P. Lioliou, M. Viberg, M. Coldrey, and F. Athley, "Self-interference suppression in full-duplex MIMO relays," Proc. 44th Asilomar Conf. Signals, Syst. Computers (ASILOMAR 2010), Pacific Grove, CA, Nov. 2010.

[8] Y.-W. Kwak, J.-H. Lee, Y.-H. Kim, and S.-C. Kim, "Precoder design and capacity analysis for multi-antenna full-duplex relay," IEICE Trans. Commun., vol.E95-B, no.7, pp.2446-2450, July 2012.

[9] J.-H. Lee and O.-S. Shin, "Full-duplex relay based on block diagonalisation in multiple-input-multiple-output relay systems," IET Commun., vol.4, no.15, pp.1817-1826, Oct. 2010.

[10] T. Taniguchi, Y. Karasawa, and N. Nakajima, "Using dirty signal How to use or not to use noise corrupted signal -," The 5th European Conf. Antennas Propagat. (EuCAP 2011), Apr. 2011.

[11] Q. H. Spencer, C. B. Peel, A. L. Swindlehurst, M. Haardt, "An introduction to the multi-user MIMO downlink," IEEE Commun. Mag., vol.42, pp.60-67, Oct. 2004.
[12] T. Fujii et al., "Radio repeater system equipped on captive balloon for mobile radio," IEICE 2012 Society Conf., B-5-32-B-5-36, Sept. 2012 (in Japanese). 